\title{
Chip-Based Radio-Frequency Tag
}

\author{
${ }^{1}$ Olga E. Zheleznikova, ${ }^{2}$ Svetlana A. Mikaeva, ${ }^{3}$ Angela S. Mikaeva, ${ }^{4}$ Alexander A. Reznik \\ ${ }^{1}$ Federal State Budgetary Educational Institution of Higher Education "National Research Mordovian State University \\ named after N.P. Ogarev" \\ ${ }^{2,3,4}$ Federal State Budgetary Educational Institution of Higher Education MIREA - Russian Technological University \\ Email: sarstf@mail.ru
}

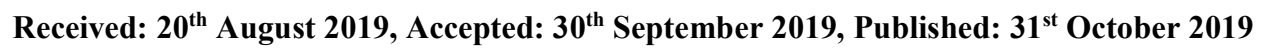

\begin{abstract}
The article is devoted to the problem associated with the operation of radio frequency tags within the UHF range in close contact with various media. The principle of RFID system operation is explained, design features and UHF RFID tag reading for metal objects are considered. They considered one of the possible options to improve the RFID chip designed to work on metal. For radio-frequency marking of metal objects, volumetric radio-frequency tags of a special design (3D tags) should be used. The quality factor of 3D tags for working on metal objects can be reduced by introducing specially selected (calculated) shunt resistors into the microcircuit design for such tags.
\end{abstract}

\section{Keywords}

Radio-frequency Tag, Flat Tag, Metal, Microcircuit, Power Supply, Amplitude-Frequency Characteristics, Reader.

\section{Introduction.}

Nowadays, radio frequency contactless identification devices (RFID) are widely used for marking and identification purposes.

Identifiers include: contactless plastic and paper cards; thin 2D self-adhesive tags; the tags made in the case (plastic) version - 3D.

In the vast majority of cases, passive RFID tags are used that do not contain any power source. Such tags are used to power the microcircuit and the responses to the surrounding space of electromagnetic energy from the electromagnetic field that trains them, produced by the reader $[1,2]$. The tags are presented on fig. 1,2 .

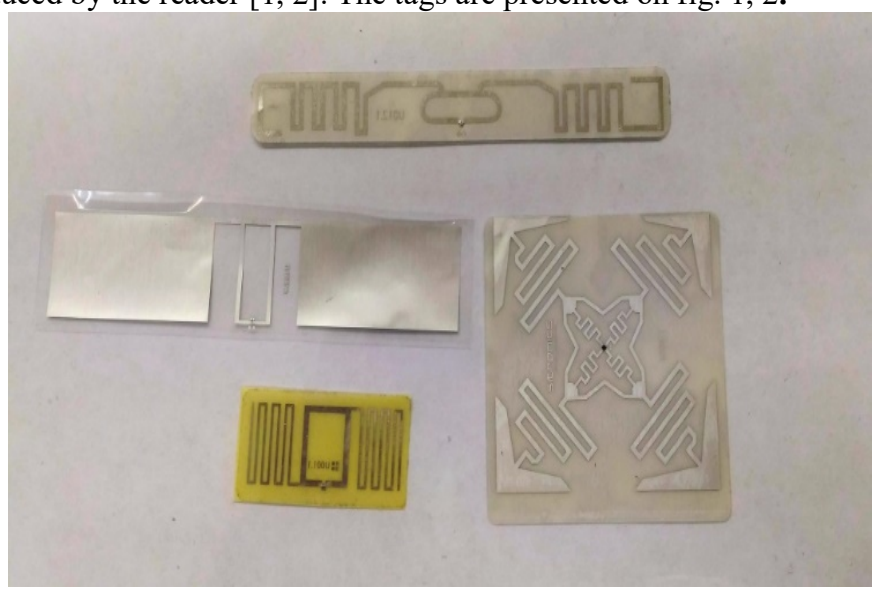

Figure 1: The Example of Flat 2D Self-Adhesive Tags (Transponders)
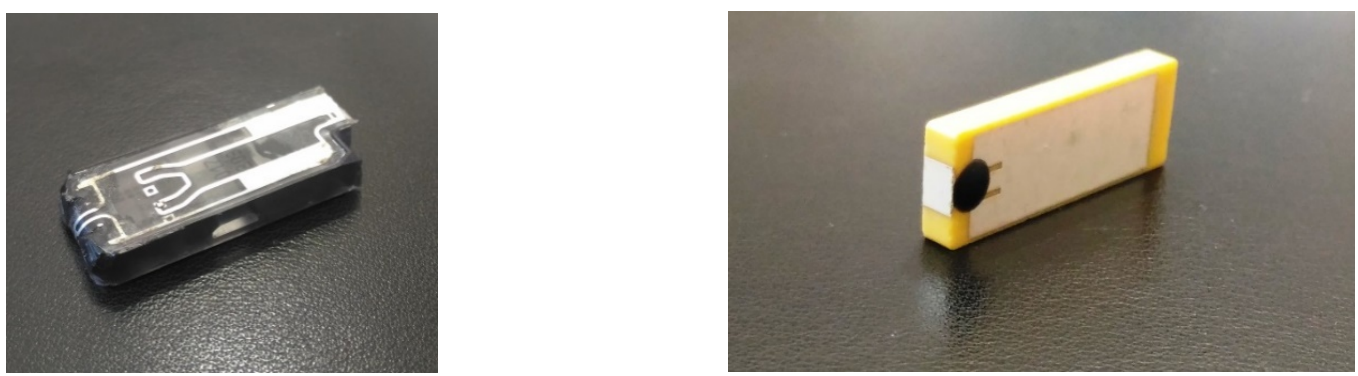

Figure 2: The Examples of 3D Volumetric Transponders

The basis of any of these constructs is a transponder, that is, a microassembly of the antenna and a radio frequency identification microcircuit mounted on it. 
In the practical application of RFID tags within the UHF band (850-990 MHz) the dependencies on the properties of the environment surrounding the tag and the distance of tag removal from a dielectric, metal or electrolytic object, an object size, the reading range and the resonant frequency of the tag + object design can vary in a specific range of values.

For example, a flat UHF radiofrequency tag ideally tuned to resonance in open space and with a long reading range, when used on real materials, may not be read by the carrier frequency of the reader - $867 \mathrm{MHz}$ due to a frequency shift (during the contact with the material).

A flat 2D UHF tag pasted onto a metal object is not always read; its range on the metal is almost "killed". An illustration of the abovementioned is shown on Fig. 3 [3].

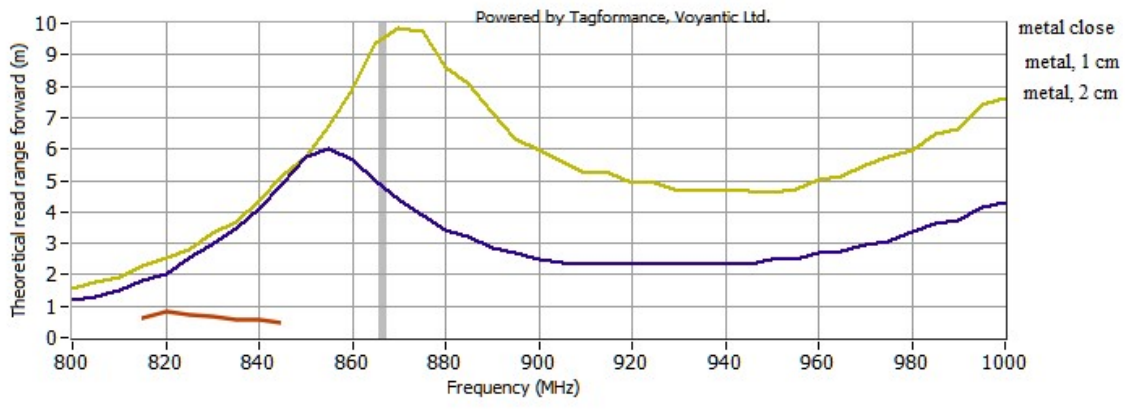

Figure 3: Amplitude-Frequency Response of the Tag Located Closely on the Metal and at the Distance of $1 \mathrm{~cm}$ and $2 \mathrm{~cm}$ from the Metal

The graphs presented in this work were performed on specialized VOYANTIC equipment intended for RFID measurements.

The measurements were carried out in the following axes: reading range - frequency. Such graphs look similar to the graphs performed in the axes: amplitude - frequency, i.e., amplitude frequency response. Therefore, in this work, similar graphs will be referred to as AFR.

Domestic chip for RFID is a complex product, it can consist of several tens and even hundreds of millions of discrete elements.

Fig. 4 shows the photo of the microcircuit topology and its main units are indicated. The microcircuit is made according to CMOS technology with the design standards of $180 \mathrm{~nm}$.

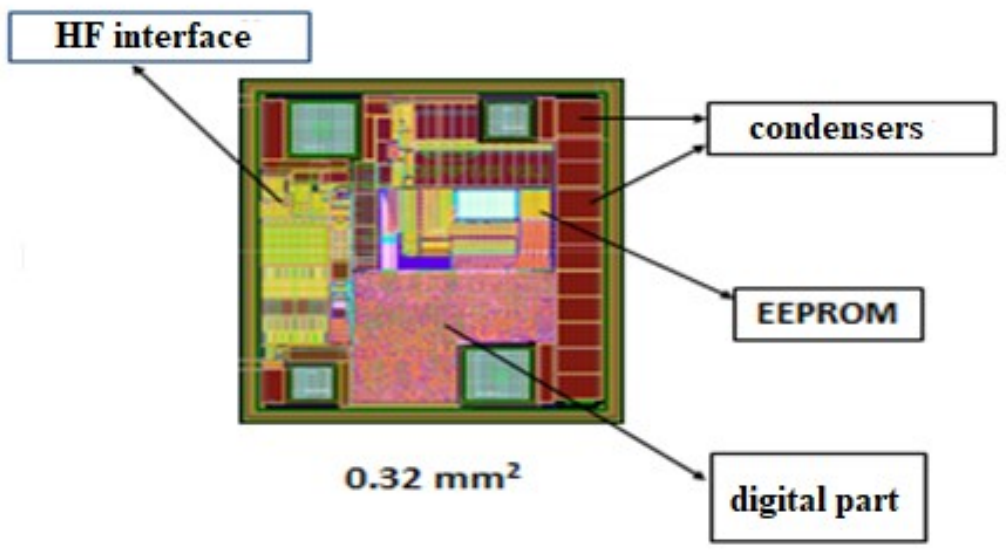

Figure 4: Internal Arrangement of RFID Microcurcuit

\section{Main Part}

The operation of RFID technology is based on the use of a reader and a tag. The reader is the source of an electromagnetic wave of a certain frequency, modulated by rectangular pulses of commands coming from the reader to the tag. The tag consists of an antenna and a chip that is able to respond to commands from the reader. The tag considered in this case refers to the so-called passive tags. It got this name because it does not contain the source of electrical energy. The tag for its work uses the energy obtained from the external electromagnetic field energy, in this case, the reader field. The principle of RFID tag operation is shown on Fig. 4., and the operation principle of the reflective UHF tag is shown on Fig. 5. 


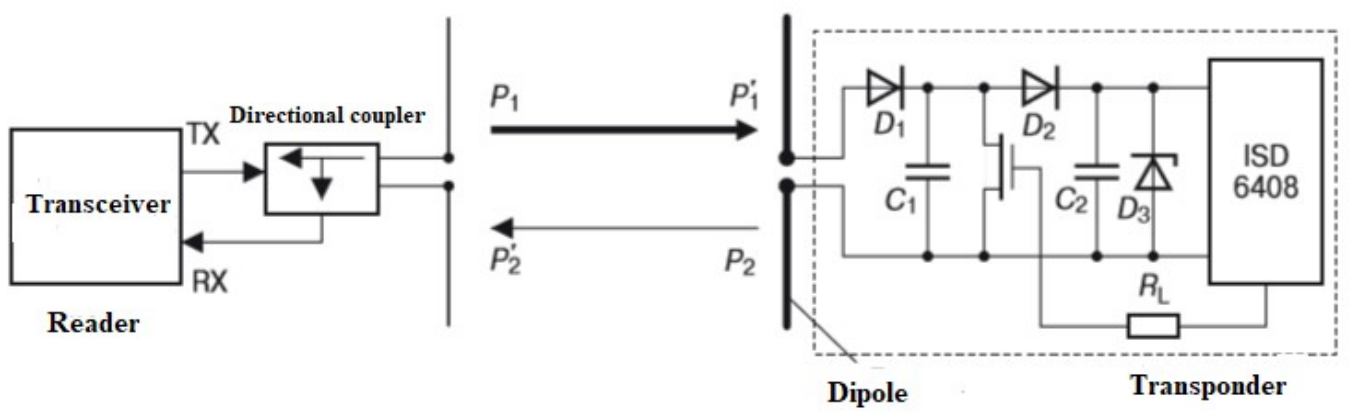

Figure 5: Reflective UHF Tag Operation Principle

The reader shown on fig. 5, generates electromagnetic radiation with the power $\mathrm{P}_{1}$, however, due to the signal weakening as the distance from the reader increases, only a small part of this radiation reaches the tag antenna. Under the influence of this radiation, high-frequency oscillations with power $\mathrm{P}_{1}$ ' occurs at the terminals of the tag antenna. The voltage rectified on the diodes $\mathrm{D}_{1}$ and $\mathrm{D}_{2}$ serves as the signal for the chip exit from the low-power mode. Schottky diodes are used here, which have an extremely low threshold voltage, low inertia and low noise level. When the voltage rectified on the diodes and accumulated in the capacitors of the microcircuit (see Fig. 4 - capacitor unit) reaches the desired value, the microcircuit will begin to respond to the reader with a set of pulses through the resistor $R_{L}$, closing and opening the electronic key located after the capacitor $\mathrm{C}_{1}$. This achieves the impedance change of the transponder input stage and the dipole antenna connected to it.

Thus, the reflectivity of the antenna changes, and the reader receiving antenna, picking up the signals reflected and modulated on the antenna of the tag, will perceive them as a stream of information from the tag. The abovementioned is shown schematically in Fig. 6, the arrow $\mathrm{S}$ denotes the tag radiation from the reader with the carrier frequency, and the arrow of the reverse (reflected) signal indicates the fact of its modulation by the tag.

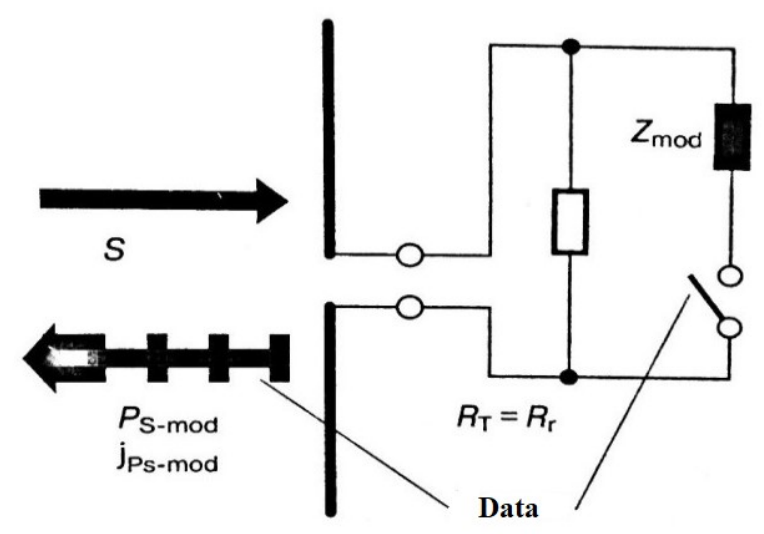

Figure 6: Tag Reflected Signal Modulation Scheme

As they noted earlier, not all types of RF tags are well suited to work on metal objects. For such objects, they use not flat, but three-dimensional tags, in which the antenna has П-shaped design deposited on a dielectric core made of various plastic materials, fused silica or langasite.

Such tags show a significant reading range on the metal $(10,20,30,40$ meters) depending on the dielectric used in them and the tag antenna configuration and are shown on Fig. 7.

This article does not consider the structural reasons for capacious tag development with a complex antenna configuration, which (unlike flat tags) can provide RFID tag operation on metal and a significant reading range.

The physics of such tag work and the features of their design are considered in other publications to which this work does not refer. 
(A)

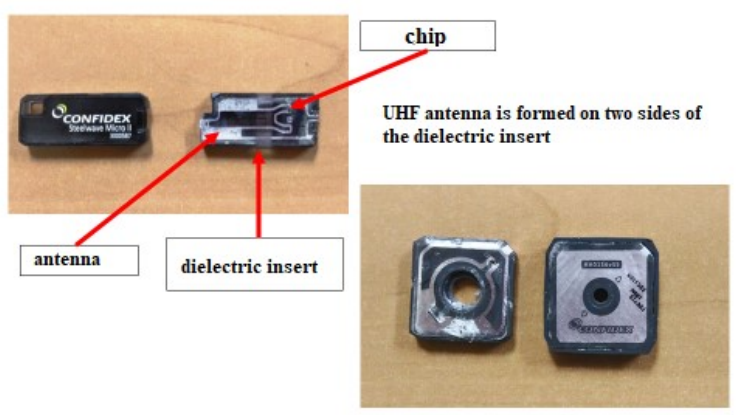

(B)

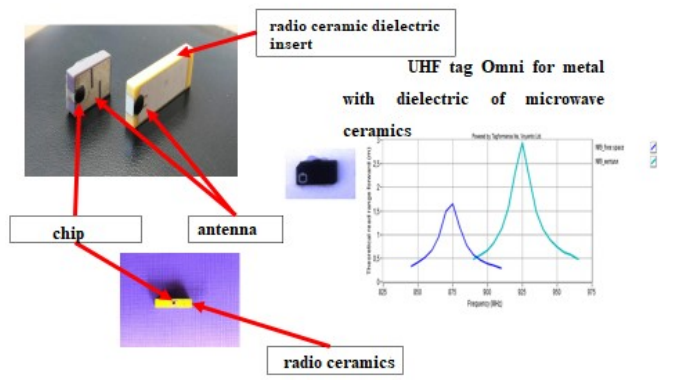

Figure 7: Tag Device for Working on Metal with Dielectric Insert Made of Polycarbonate (A) and with Dielectric Strip Made of Radio Ceramics (B).

It was found that when you work on the metal of the abovementioned tags, their amplitude frequency response becomes very high-quality, the resonant frequency during the contact with materials of various shapes and sizes also deviates left or right from the required carrier frequency of the reader $(865 \mathrm{MHz}$ or $920 \mathrm{MHz}$ ) at 5-10 MHz. The reading range of the tag at the carrier frequency is nullified as the result of this effect.

Such high-quality tags are also very difficult to assemble serially and with strictly fixed parameters. The slightest deviation of the antenna or microcircuit location inside the tag leads to the deviation from the design resonant frequency and a sharp drop of the reading range, which is shown on Fig. 8 - 9.

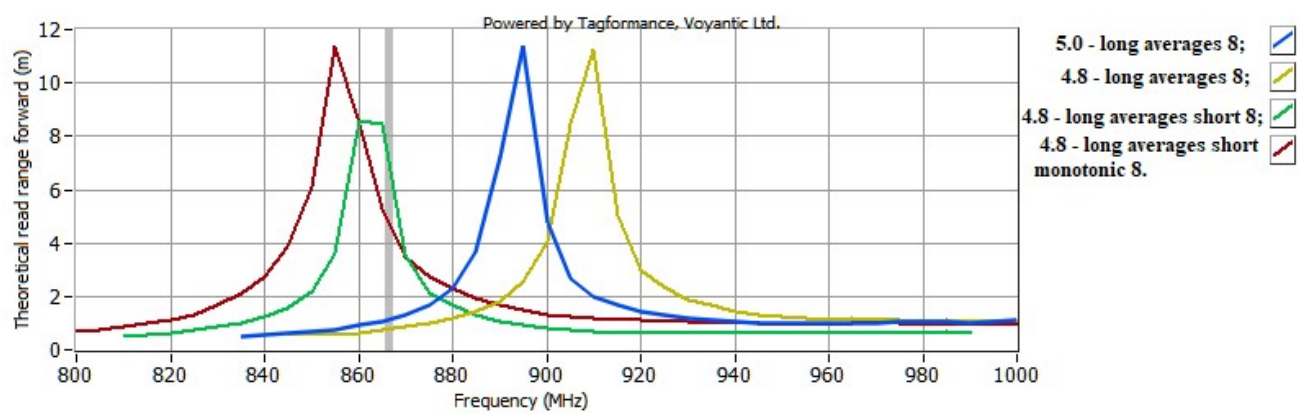

Figure 8: Scatter of the Tag Resonant Frequency Parameters for Metal with Minor Changes in Antenna Size during the Product Assembly.

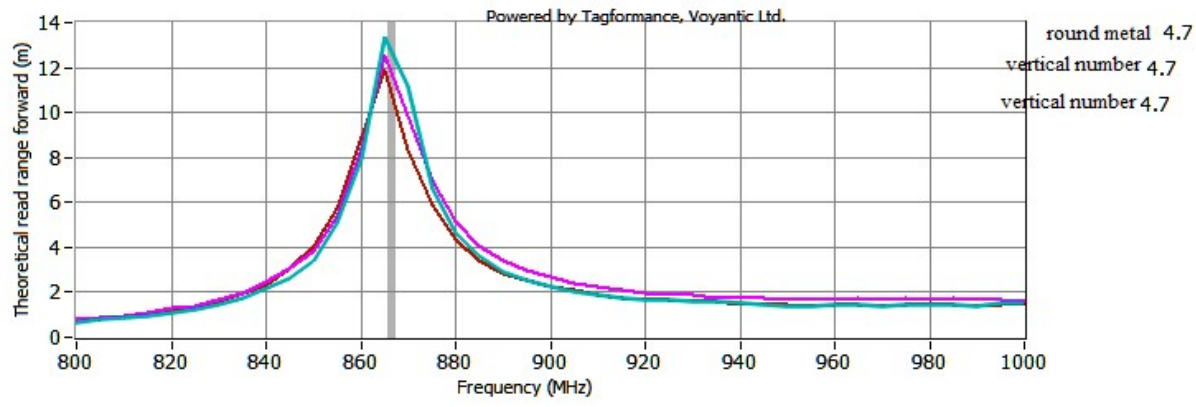

Figure 9: The Required Range of Tag Parameters for Metal

The graphs show that the deviation from the resonant frequency $(867 \mathrm{MHz}$, indicated by a gray vertical marker) at least of $15 \mathrm{MHz}$ in any direction from the resonant frequency leads to readout reduction by two or more times.

An obvious action to alleviate the situation is the case of introducing changes to the input impedance of the microcircuit for metal applied tags, which will allow a reasonable quality factor reduction of the antenna-microcircuit loop and expand the bandwidth by a little.

In other words, the thing is about the creation of special microcircuits intended only for tags working on metal and differing with their structural properties from microcircuits needed for flat tags $[4,5]$.

Overseas they have recently managed to solve this problem [6 - 9]. Corresponding changes were made in the U CODE8 
microcircuit. Thus the AFR of the metal applied tags based on this microcircuit acquired a very acceptable form for users. The shelf of constant reading range (25 meters) has become wider (Fig. 10).

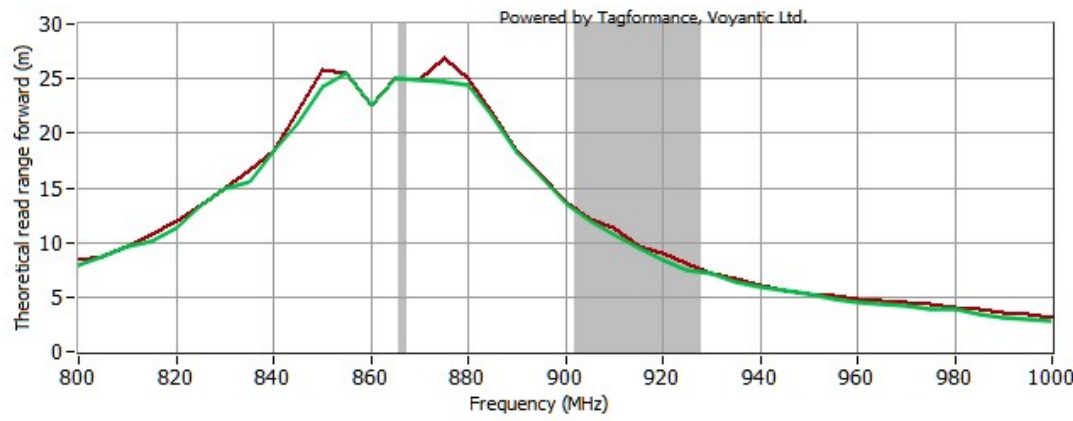

earthy black width earthy gray width

Figure 10: AFR of the Metal Applied Tag Based on the U Code8 Chip

Due to the fact that the domestic UHF chip intended for working on metals will also need to be developed [10], an attempt was made in this paper to conduct an experiment with the aim of expanding the passband of the tag due to an acceptable decrease of the input circuit quality factor for the UHF tag.

This should ensure a guaranteed stable reading range in the band $\mathrm{F}_{\text {res }}$ plus / minus $15 \mathrm{MHz}$.

As a first step, it is proposed to add shunt resistance of a certain nominal value to the composition of the input resistance of the microcircuit. This upgrade will expand the bandwidth of the tag.

The standard domestic UHF microcircuit for flat tags was taken as the object of study, on the basis of which a thin tag ( $2 \mathrm{~mm}$ ) applied on metal was made, as shown on Fig. 11. This tag did not have a significant range, which is usually natural for volume tags with the thickness of $5 \mathrm{~mm}$ to $10 \mathrm{~mm}$.

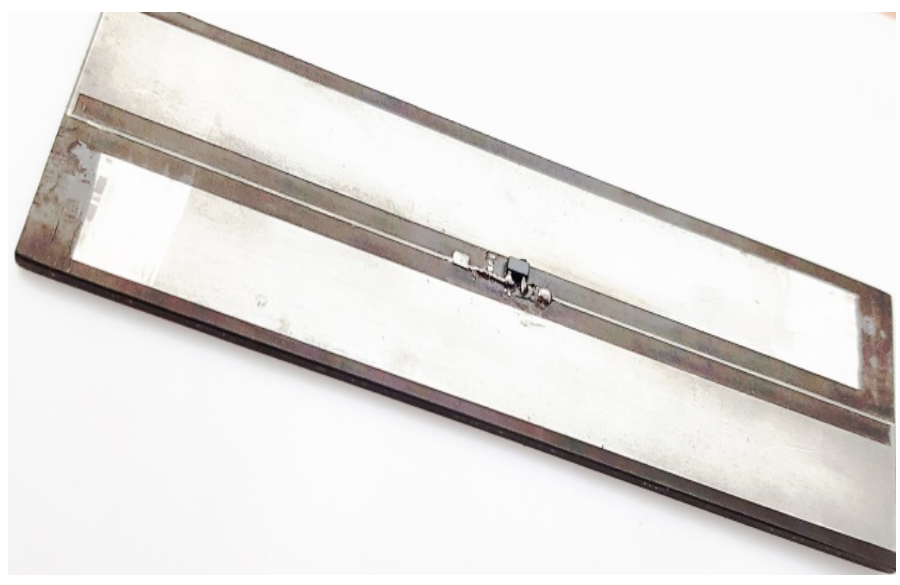

Figure 11: Metal Tag Photo based on Domestic Microcircuit

For the abovementioned label, the AFR was obtained (Fig. 12) with the following parameters: $\mathrm{F}_{\text {res }}=920 \mathrm{MHz}$; R - tag reading range $-5 \mathrm{~m}$.

The input impedance $(Z)$ of the applied microcircuit at the frequency of $915 \mathrm{MHz}$ had a complex value of 12.5 - j277. In the original label, the impedances of the microcircuit and antenna were complexly conjugated. In this tag, the input of the microcircuit was shunted several times by parallel resistances of $5 \mathrm{kOhm}, 10 \mathrm{kOhm}, 22 \mathrm{kOhm}, 33 \mathrm{kOhm}$. After the research, the experimental results were obtained shown on Fig. 13. 


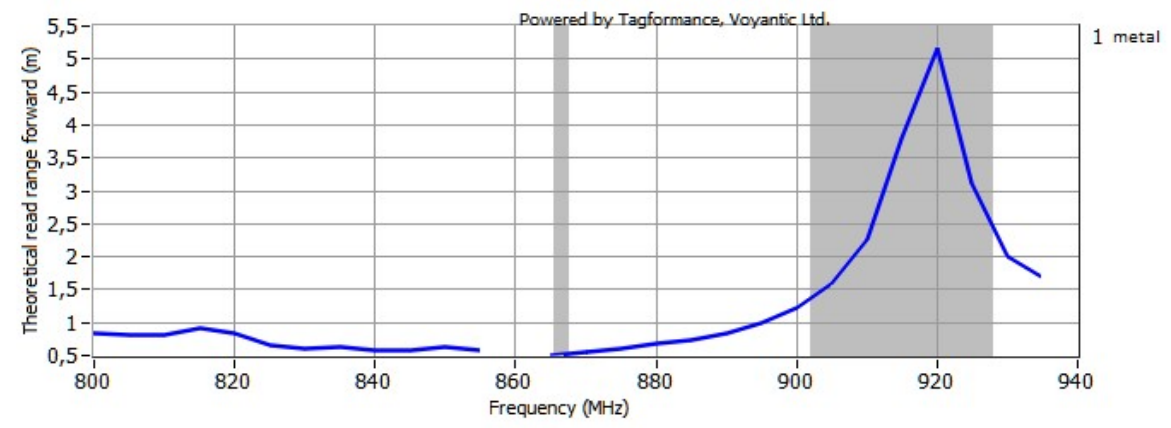

Figure 12: AFR Tags for Metal based on Domestic Microchip

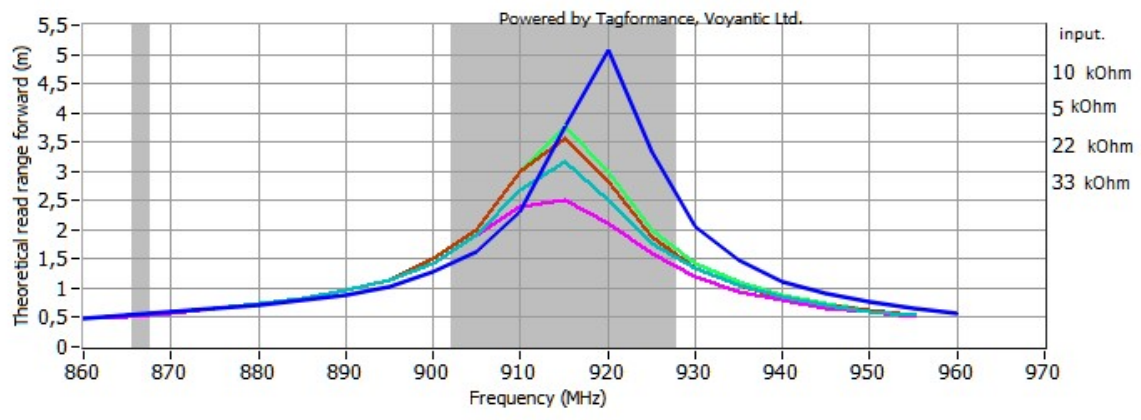

Figure 13: AFR Tags at Different Values of Shunting Resistance

\section{Summary}

It was found that the optimal shunt resistance for this type of chip is the resistance of $33 \mathrm{kOhm}$. After its use, the reading range decreased from $5.5 \mathrm{~m}$ to $3.5 \mathrm{~m}$ (1.57 times). But the bandwidth has almost doubled - from $10 \mathrm{MHz}$ to 19 $\mathrm{MHz}$.

The shift to the left of the resonant frequency of the tag can be easily restored to $920 \mathrm{MHz}$ due to a slight decrease of the upper emitter length of the tag antenna.

\section{Conclusions}

Flat traditional RFID tags cannot be used for radio-frequency marking of metal objects, since they cannot be read out in close contact with metals.

For radio-frequency marking of metal objects, volumetric radio-frequency tags of a special design (3D tags) should be used.

3D tags show a significant reading range during operation on metal objects.

3D tags during operation on metal objects demonstrate high-quality AFR in the following axes: frequency - reading range, which for the conditions of their use and production is more a disadvantage than an advantage.

The quality factor of $3 \mathrm{D}$ tags for working on metal objects can be reduced by introduction of specially selected (calculated) shunt resistors for such tags into the microcircuit design.

The input impedance of microcircuits used in 3D tags for working on metal should be calculated based on the requirements of a bandwidth of at least $20 \mathrm{MHz}$ when setting a tag on metal.

Chips for flat RFID tags should not be recommended for use in the tags for working on metal objects.

\section{References}

1. Mikaeva S.A., Brysin A.N., 2018. Electrical Engineering. - Kazan: Buk: 228.

2. Larionov M.Yu., 2017. Theoretical substantiation of the reasons for changing the read parameters of the RF tags in the UHF range when they come in contact with dense external media. Collection of reports of the 60th scientific-practical conference. MIPT.

3. Babenko V.P., Bityukov V.K., Kuznetsov V.V., Simachkov D.S. 2018. Modeling of static and dynamic losses in MOSFET keys. Russian Technology Journal: 6 (1): 20-39.

4. Mikaeva, S.A., Mikaeva, A.S., 2017. Protective Coatings. Glass and Ceramics (English translation of Steklo i Keramika): 74(3-4):144-146.

5. Boichuk, M.I., Zheleznikova, O.E., Mikaeva, S.A, Mikaeva, A.S., 2019. Metal-ceramic enclosure for surface mounting. International transaction journal of engineering management. Applied sciences. Technologies: 10: 551-558.

6. Finkenzeller, Klaus, 2008. Reference RFID. Dodeka-XXI: 488 p. 
7. Wolfram, G., Gampi, B., Gabriel, P., 2008. The RFID Roadmap: The Next Steps for Europe. Springer Science \& Business Media: 112-118.

8. Kivshar, Yu. S., 2015. From metamaterials to metasurfaces and metadevices. Nanosystems: physics, chemistry, mathematics: 6 (3): 346-352.

9. Hasan, N., 2014. Roadmap for RFID Implementation in Libraries: Issues and Challenges. International Journal of Information, Library and Society: 3(1): 65-71.

10. Grigoryev, P.V., 2016. Osobennosti tekhnologii RFID i ee primenenie [Features of RFID technology and its application]. Molodoy uchenyy. [Young scientist]: 11: 317-322. 